\title{
Erratum to: The sequestrate genus Rossbeevera T.Lebel \& Orihara gen. nov. (Boletaceae) from Australasia and Japan: new species and new combinations
}

\author{
Teresa Lebel • Takamichi Orihara • Nitaro Maekawa
}

Published online: 11 August 2011

(C) Kevin D. Hyde 2011

\section{Erratum to: Fungal Diversity}

$$
\text { DOI 10.1007/s13225-011-0109-x }
$$

We wish to correct a spelling error in the original publication for a newly described genus honouring the New Zealand mycologist Ross Beever. The correct spelling is Rossbeevera $\mathrm{T}$. Lebel \& Orihara gen. nov. A list of the species names follows.

Rossbeevera bispora (B.C.Zhang \& Y.N.Yu) T.Lebel \& Orihara comb. nov.

The online version of the original article can be found at http://dx.doi. org/10.1007/s13225-011-0109-x.

\section{T. Lebel $(\bowtie)$}

National Herbarium of Victoria,

Royal Botanic Gardens Melbourne,

Private Bag 2000, Birdwood Ave,

South Yarra, VIC 3141, Australia

e-mail: teresa.lebel@rbg.vic.gov.au

\section{T. Orihara}

The United Graduate School of Agriculture Sciences,

Tottori University,

4-101 Koyama-cho-minami,

Tottori 680-8553, Japan

\section{N. Maekawa}

Fungus/Mushroom Resource and Research Center,

Faculty of Agriculture, Tottori University,

4-101 Koyama-cho-minami,

Tottori 680-8553, Japan

\section{Present Address:}

T. Orihara

Kanagawa Prefectural Museum of Natural History,

499 Iriuda,

Odawara-shi, Kanagawa 250-0031, Japan
Rossbeevera eucyanea Orihara sp. nov.

Rossbeevera griseovelutina Orihara sp. nov.

Rossbeevera mucosa (Petri) T.Lebel comb. nov.

Rossbeevera vittatispora (G.W.Beaton, Pegler \& T.W.K. Young) T.Lebel comb. nov.

Rossbeevera westraliensis T. Lebel sp. nov. 